\title{
BMJ Open Quality Virtual phone clinics in orthopaedics: evaluation of clinical application and sustainability
}

Raj Pradhan, ${ }^{1}$ Wouter Peeters (D) , ${ }^{1,2}$ Sara Boutong, ${ }^{1}$ Chris Mitchell, ${ }^{1}$ Rahul Patel, ${ }^{1}$ Rad Faroug, ${ }^{1}$ Mark Roussot (i) ${ }^{1}$

To cite: Pradhan $\mathrm{R}$, Peeters W, Boutong S, et al. Virtual phone clinics in orthopaedics: evaluation of clinical application and sustainability. BMJ Open Quality 2021;10:e01349. doi:10.1136/ bmjoq-2021-001349

Received 18 January 2021 Accepted 5 July 2021

\section{Check for updates}

(C) Author(s) (or their employer(s)) 2021. Re-use permitted under CC BY-NC. No commercial re-use. See rights and permissions. Published by BMJ.

${ }^{1}$ Department of Trauma and Orthopaedics, University

College London Hospitals NHS

Foundation Trust, London, UK

${ }^{2}$ Department of Orthopaedics, University Hospital Antwerp, Edegem, Belgium

Correspondence to Mr Mark Roussot; mark.roussot@nhs.net

\section{ABSTRACT}

Aims Adoption of virtual clinics has been accelerated by the COVID-19 pandemic and they will continue to form an integral part of healthcare delivery. Our objective was to evaluate virtual clinics in orthopaedic practice and determine how to use them effectively and sustainably. Methods We surveyed 100 consecutive patients participating in orthopaedic virtual phone clinic (VPC) at an academic hospital to evaluate patient satisfaction against face-to-face (F2F) consultations and obtain suggestions for improving patient experience, and we surveyed 23 clinicians who conducted orthopaedic VPCs in 2020. Data were correlated with clinic outcomes, reason for consultation, diagnosis, patient age and clinician grade. Consultation duration, clinician-associated costs and reimbursement were analysed. Significance was tested using two-tailed Student's t-test and Fisher's exact test. Results Patient satisfaction (out of 5) for VPC was significantly lower than F2F (4.1 vs $4.5, p=0.0003$ ), and a larger proportion of VPC scored $<3$ compared with F2F (11\% vs $2 \%)$. Higher VPC scores were associated with appointments for delivering results and where patients felt clinical examination was not needed. Patients suggested introducing video capability, adhering to appointment time and offering the choice of VPC or F2F. Mean clinician satisfaction scores for VPC were 4.3/5 and suggested indications for VPC included: routine surveillance, communication of results, discussing/ consenting for surgery and vulnerable patients. Integrating video, providing private rooms and offering patients time intervals for VPC were recommended. Current National Health Service VPC structures uses greater clinician resources and generates lower reimbursement than $\mathrm{F} 2 \mathrm{~F}$ consultations, resulting in $11.5 \%$ reduction in reimbursement.

Conclusion VPC plays a valuable role when clinical evaluation has been performed or considered not necessary. Offering the choice of VPC or F2F, adding video capability and providing a time interval for VPC may reduce resource use and increase satisfaction. We recommend renegotiating VPC tariffs and cost-neutral modifications of clinic structure.

\section{INTRODUCTION}

The delivery of healthcare from a remote location using integrated computer and telecommunication technology, referred to as telemedicine, ${ }^{12}$ has been studied in contexts such treating patients with asthma ${ }^{3-5}$ or with cancer, ${ }^{67}$ but its application in orthopaedics prior to the COVID-19 pandemic was largely limited to the experience of remote orthopaedic outreach clinics. $^{8-10}$ Despite initial infrastructure and training requirements, these studies have demonstrated that remote clinics are not only acceptable to patients and clinicians, but also cost-effective, as they reduce travel costs and absence from work. ${ }^{1112}$ Telemedicine and virtual clinics will continue to play a role in healthcare, as prolonged or intermittent social distancing may be necessary for the foreseeable future ${ }^{13}$ and patients will benefit from diversification of methods of service delivery. ${ }^{14}$

Although it recently has been shown that virtual clinics can be rapidly implemented, ${ }^{15}$ there still is limited guidance on telemedicine in the orthopaedic context, especially on virtual phone clinics (VPCs). Those guidelines that do exist are early in development. ${ }^{16}$ For VPCs to provide a meaningful role, we need to determine the most effective methods for using them sustainably and identify appropriate patient selection criteria.

The COVID-19 19 pandemic stimulated rapid adoption of virtual orthopaedic clinics throughout the UK to enable social distancing and protect vulnerable patients from unnecessary exposure to the hospital environment. These VPCs are performed entirely via telephone, with clinicians in a private and quiet space with computer access and patients in their own homes. Clinicians would perform these clinics in clinic rooms previously used for face-to-face clinics (F2F). The universal rollout of VPCs across the country in light of the pandemic evidences the potential for widespread use of VPCs in the future, but it is important they are evaluated for consideration of further use postpandemic.

Our objective was to evaluate the role of virtual clinics in orthopaedic practice and determine how to use them effectively and 
Table 1 Patientand clinician satisfaction questionnaires

(A) Patient 3-point questionnaire

1. How would you rate the concept of a telephone/virtual clinic (not the person you spoke to) on a scale from 1 to 5 , where 1 is very poor, 2 is poor, 3 is neutral, 4 is good and 5 is excellent?

2. How would you rate your last face-to-face appointment (not the person you spoke to) on a scale from 1 to 5 , where 1 is very poor, and 5 is excellent?

3. How can we make a telephone/virtual clinic better?

(B) Clinician satisfaction questionnaire

1. How would you rate the concept of a telephone/virtual clinic on a scale from 1 to 5 , where 1 is very poor, 2 is poor, 3 is neutral, 4 is good and 5 is excellent

2. Would you be willing to do telephone clinics in future?

3. Which patients would be best suited to telephone clinics?

4. How could telephone/virtual clinics be improved?

sustainably by (1) comparing virtual and F2F clinics in terms of patient and clinician satisfaction in orthopaedic consultations and (2) evaluating the costs associated with VPCs in an National Health Service (NHS) Foundation Trust.

\section{METHODS}

\section{Setting}

This three-arm study evaluated VPCs in orthopaedic clinics at an academic hospital in the UK, between May and June 2020. There was complete adherence to our intervention and the entirety of our orthopaedic department switched to using VPC (with exceptions for patients where F2F consultation was absolutely necessary) due to the COVID-19 pandemic.

\section{Study procedure}

Patients gave verbal consent for the postclinic telephone interview, which would then be conducted by a study author not involved in the care of that patient (RP). All data were anonymised and collected on a passwordprotected computer. Patient satisfaction was assessed by a three-point questionnaire in which patients were asked to give both their VPC and the preceding F2F a rating out of 5 , where 1 is very poor, 3 is neutral and 5 is excellent, and to provide suggestions for improving VPCs (table 1A). Additional data such as patient characteristics (age, sex, consult type, consult subspecialty), VPC actions, VPC outcomes and duration of VPC were collected using our local electronic health records system. Exclusion criteria included patients who were not contactable (five patients) and patients who required language translation services (two patients).

The clinician perception was assessed by an anonymised four-questionnaire survey (table 1B) using Google Surveys and was completed by 23 clinicians (13 T\&O Consultants, 9 T\&O Registrars/Fellows and 1 physiotherapist extended practitioner) who had performed VPCs during the study period.

The cost-effectiveness analysis of VPCs versus F2F involved a review of respective clinic structures and compared consultant time required, registrar time required, duration of consultation and the current local reimbursement structure for clinic provision which is based on the guideline figures set by NHS UK. ${ }^{17}$

\section{Analysis}

Data analyses were conducted with Microsoft Excel and significance testing was performed with the two-tailed Student's t-test for means and Fisher's exact test for proportions where relevant. One patient was excluded from age analysis comparisons as the patient was 1 year old, however, evaluation of clinic satisfaction by the parents was included in all other data sets.

The clinician score was recorded with a 5-point Likert scale (1-5), while the patient feedback scores were recorded with a 10-point Likert scales (1-10). For ease of comparison, we converted the 10-point scale to a 5-point scale using a simple, validated arithmetic method ${ }^{18}$ whereby the 10-point score was adjusted by dividing the score by 2. Scores of 0.5 and 1 in the adjusted 10-point scale were equivalent to 1 in the 5 -point scale (very poor); scores of 2.5 and 3 in the adjusted 10-point scale were equivalent to 3 in the 5-point scale (neutral); and scores of 4.5 and 5 in the adjusted 10-point scale were equivalent to 5 in the 5-point scale (excellent).

A score of 3 or more out of 5 was considered a 'neutral or satisfied' score; a score of 1 or 2 was considered a 'dissatisfied' score.

\section{Patients and public involvement}

Patients and the public were not involved in this study, including data collection, analysis and interpretation.

\section{RESULTS}

\section{Patient satisfaction}

The 100 patients who participated in the study were predominantly female $(62 \%)$ and had a mean age of 52.8 years (SD 18.7). Consultations were conducted in elective hip, knee and foot and ankle clinics $(79 \%)$ and fracture clinic $(21 \%)$. Call duration was measured for 44 out of 
Table 2 Satisfaction scores for VPC and F2F for various patient groups

\begin{tabular}{|c|c|c|c|c|}
\hline & \multirow[b]{2}{*}{ No of patients } & \multicolumn{2}{|c|}{ Mean satisfaction score } & \multirow[b]{2}{*}{$P$ value } \\
\hline & & VPC & F2F & \\
\hline All patients & 100 & 4.1 & 4.5 & $<0.001$ \\
\hline \multicolumn{5}{|l|}{ Dissatisfied patients $(<3 / 5)$} \\
\hline VPC & 11 & 2.0 & 3.5 & $<0.001$ \\
\hline $\mathrm{F} 2 \mathrm{~F}$ & 2 & 3.8 & 2.3 & 0.051 \\
\hline \multicolumn{5}{|l|}{ Operative status } \\
\hline Preoperative & 44 & 4.2 & 4.5 & 0.057 \\
\hline Postoperative & 32 & 4.1 & 4.5 & 0.013 \\
\hline Non-operative management & 24 & 4.0 & 4.5 & 0.033 \\
\hline \multicolumn{5}{|l|}{ Reason for consult } \\
\hline Listed for surgery & 12 & 4.0 & 4.8 & 0.088 \\
\hline Arrange investigation & 7 & 4.0 & 4.9 & 0.090 \\
\hline Communicate results & 10 & 4.2 & 4.7 & 0.195 \\
\hline Refer to therapy & 19 & 3.8 & 4.5 & 0.043 \\
\hline Routine follow-up & 52 & 4.2 & 4.5 & 0.008 \\
\hline \multicolumn{5}{|l|}{ Consult outcome } \\
\hline Discharged from clinic & 21 & 4.4 & 4.4 & 0.901 \\
\hline Patient initiated follow-up & 12 & 4.0 & 4.5 & 0.160 \\
\hline New follow-up appointment booked & 67 & 4.0 & 4.5 & $<0.001$ \\
\hline
\end{tabular}

F2F, face to face; VPC, virtual phone clinic.

100 VPCs (44.0\%) and an average phone call duration of was $8.9 \mathrm{~min}$ (SD 3.047) was calculated.

Overall, the mean patient satisfaction score out of 5 was high (4.3, SD 0.839). However, VPC scored significantly lower than F2F clinics (4.1 (SD 0.978) vs 4.5 (SD 0.610), $\mathrm{p}<0.001$ ). Analysis of the operative status, reason for consultation and consultation outcome, summarised in table 2, demonstrated that patients being managed nonoperatively and being followed up after surgical intervention reported higher satisfaction scores for F2F versus VPC, but no significant differences were noted when listing for a minor procedure, arranging investigations or communicating results. When referring to therapies F2F consultation scored significantly higher than VPCs (3.8 (SD 0.879) vs 4.5 (SD 0.752); $\mathrm{p}=0.043$ ).

VPC showed a larger distribution of lower scores with $11 \%$ of virtual consultations scoring $<3$ vs only $2 \%$ of $\mathrm{F} 2 \mathrm{~F}$ consultations scoring $<3$ (figure 1). Of the 89 neutral or satisfied patients who scored their VPC clinic $\geq 3$, the difference in satisfaction score with F2F clinics was not significant (4.4 for VPC (SD 0.635) vs 4.6 (SD 0.495) for $\mathrm{F} 2 \mathrm{~F} ; \mathrm{p}=0.687$ ). The 11 patients with dissatisfied scores for VPC had a large difference in mean satisfaction scores for VPC vs F2F; 2 (SD 0.671) vs 4.4 (SD 0.757) respectively $(p<0.001)$. VPCs scored relatively well when conducted for communication of results (4.2; SD 0.888) and for routine follow-up appointments (4.2; SD 0.483). Patients were divided into age groups and compared but no statistically significant differences were found, though F2F appointments scored higher in all age groups.
Patients with 'satisfied' scores felt that VPCs were 'convenient' or 'safer' (20 patients). Those with 'dissatisfied' scores would have preferred a clinical examination (12 patients) or felt the communication was 'ineffective' or 'not reassuring' (9 patients, (table 3A). Patients recommended including video capability, having a dedicated time for the VPC consultation and offering the choice of VPC or F2F consultation (table 4A).

\section{Clinician satisfaction}

The mean clinician satisfaction score both for consultants and registrars/fellows was high, 4.2 (SD 1.24) and 4.4 (SD 0.53), respectively $(\mathrm{p}=0.632)$. Of the 23 clinicians surveyed, $22(95.7 \%)$ were willing to do VPCs in

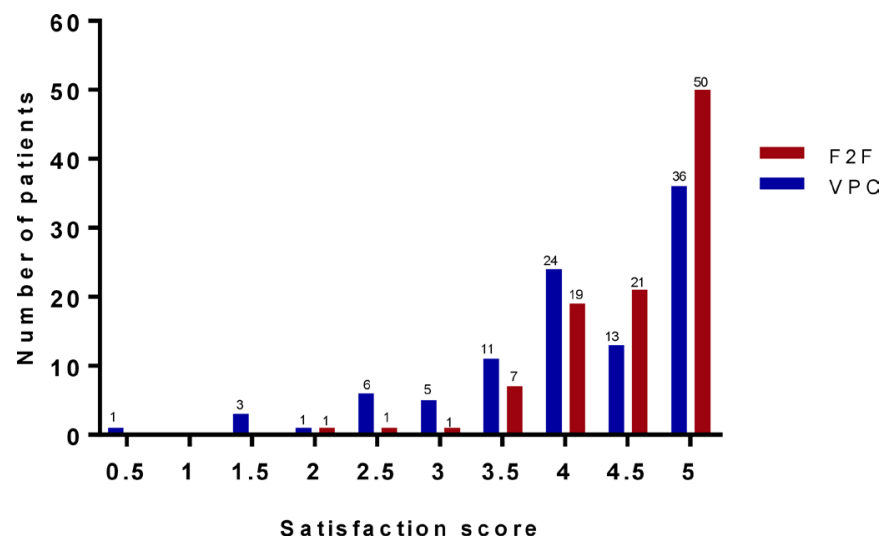

Figure 1 Distribution of patient satisfaction scores (out of 5). F2F, face to face; VPC, virtual phone clinic. 
Table 3 Patients' and clinicians' feedback on VPC

(A) Patients' feedback

\begin{tabular}{|c|c|}
\hline Advantages & Disadvantages \\
\hline Convenient & Missing clinical assessment \\
\hline \multirow[t]{2}{*}{ Safer } & Not reassuring \\
\hline & Ineffective communication \\
\hline \multicolumn{2}{|l|}{ (B) Clinicians' feedback } \\
\hline Advantages & Disadvantages \\
\hline Convenient & $\begin{array}{l}\text { Clinical assessment not } \\
\text { possible }\end{array}$ \\
\hline Patient centred & $\begin{array}{l}\text { Duplication of work if F2F } \\
\text { assessment required }\end{array}$ \\
\hline Good for triaging patients & $\begin{array}{l}\text { Current methods of } \\
\text { documenting inefficient }\end{array}$ \\
\hline
\end{tabular}

Facilitates information sharing/

management decision

F2F, face to face; VPC, virtual phone clinic.

the future. Clinicians noted that VPCs were convenient, patient-centred and facilitated information sharing. The lack of clinical assessment was highlighted as the main concern (tables 3B and 4B).

Clinicians were asked how suitable they felt various consult types were for VPC. The majority of the 23 clinicians felt 'routine surveillance' (18; 78.3\%) and 'communicating results' $(20 ; 87.0 \%)$ were appropriate for VPC. 'Early post-operative follow-up' (3; 13.0\%), 'listing for surgery' $(3 ; 13.0 \%)$ and ' new/changing symptoms' (1; $4,3 \%$ ) were considered unsuitable for VPC.

No cases were noted to have a 'missed diagnosis'.

\section{Cost assessment}

The calculation of VPC cost-effectiveness was based on the reimbursement structure for the current clinic provision in our hospital, which in turn is based on the guideline figures set by NHS UK. ${ }^{17}$ Our hospital provides services and bills NHS UK through the local Clinical Commissioning Group (CCG). Tariffs before the COVID-19 pandemic were set at £206.01 for F2F new patient appointments, $£ 81.65$ for F2F follow-up appointments, and £31.32 for VPCs follow-up appointments. This equates to a $£ 50.33$ $(61.64 \%)$ difference per appointment for VPC compared with F2F. Through the duration of our study, VPC and F2F follow-up appointments had been temporarily tariffmatched to encourage reducing patient contact during the COVID-19 pandemic.

Despite a mean telephone call time of $8.9 \mathrm{~min}$, the total time of a VPC was found to be equivalent to that expected of an F2F follow-up consultation. ${ }^{10}$ Additionally, the process of triaging patients to F2F or VPC took on average 1 hour of consultant time per 3 hours clinic, equating to $£ 77.75$ extra work cost $(123.8 \%)$.

If tariffs were to return to prepandemic rates in future, the hospital would attract $61.6 \%$ less in tariff for each VPC compared with an F2F follow-up consult even if triaging time was not taken into account. Given an orthopaedic clinic currently consists of $40 \% \mathrm{~F} 2 \mathrm{~F}$ new patient appointments, 30\% F2F follow-up appointments and 30\% VPC follow-up appointments, this would involve a loss of reimbursement of approximately $11.5 \%$ per 3 hours clinic (figure 2). This is an organisational barrier to readiness and could make VPCs unsustainable should the prepandemic tariffs return.

\section{DISCUSSION}

This study demonstrated that F2F consultations remain the preferred method of interaction for orthopaedic follow-up clinics for patients and clinicians. However, VPC plays a useful role in specific scenarios such as when clinical examination has already been performed or

Table 4 Patients' and clinicians' suggestions for improving VPCs

(A) Patients' suggestions

\begin{tabular}{|c|c|}
\hline Infrastructure & Process \\
\hline Integrate video capability & Create a dedicated time slot \\
\hline $\begin{array}{l}\text { Create option to share and discuss } \\
\text { imaging/scans }\end{array}$ & Create option for F2F \\
\hline
\end{tabular}

(B) Clinicians' suggestions

\begin{tabular}{lll} 
Infrastructure & Process & Other \\
Integrate video capability & Improved triage involving patients & Preappointment info sheet with questions \\
Expansion of dictaphones & $\begin{array}{l}\text { Time windows versus exact times for } \\
\text { calling patients }\end{array}$ & $\begin{array}{l}\text { Tell patient to expect call from a private } \\
\text { number }\end{array}$ \\
Dedicated quiet rooms & Patient led discharge & \\
Clinical Nurse Specialist help & More flexibility in clinic times & \\
Virtual waiting room & Categorise appointments & \\
Mobile application & & \\
\hline
\end{tabular}

F2F, face to face; VPC, virtual phone clinic. 


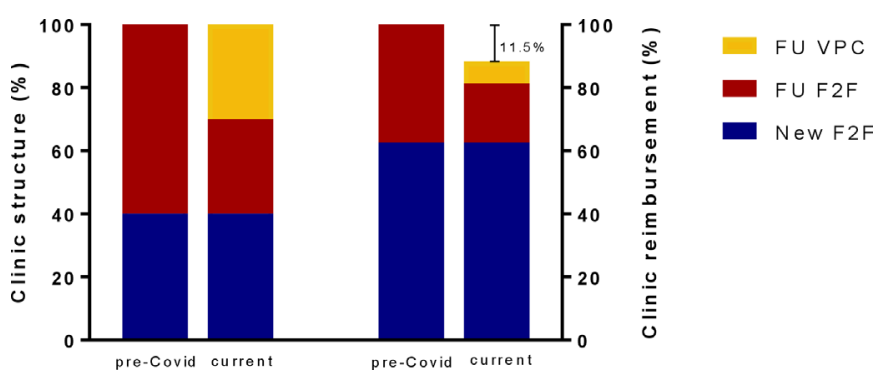

Figure 2 Cost assessment. the left two bars show the difference in clinic structure between the pre-COVID and current situation (in \%). The right two bars show the effect on the clinic reimbursement (in \%, normalised to the pre-COVID reimbursement). F2F, face to face; FU, follow-up; VPC, virtual phone clinic.

deemed not to be necessary by patients or clinicians, for routine follow-up consultations and for communication of results. We identified three simple interventions for VPC that may reduce the resources required and increase utility and satisfaction rates: offering patients the choice of VPC or F2F, adding video capability and providing a time interval for the consultation. However, for long-term sustainability, renegotiating the tariff for VPC along with cost-neutral modifications of the clinic structure may be required.

Unsurprisingly, both patients and clinicians considered the inability to conduct a clinical examination as a significant disadvantage to VPC and this is reflected in the overall preference for F2F consultations. This preference has been investigated in a previous study into the acceptability of virtual follow-up clinics for elective hip and knee replacements, and the reasons for this preference are multifactorial. ${ }^{19}$ The addition of video capability, recommended by both patients and clinicians, could meet challenges such as technical support, quality of clinical assessment, safety, privacy and even the potential for litigation that may arise if something goes wrong ${ }^{20}$ On the other hand, patients could, in conjunction with verbal communication, demonstrate clinical features such as improvements in wound healing, joint range of motion and gait following uncomplicated, elective procedures, for example. Evidence for video consultations is sparse but that which does exist suggests they are safe and effective, ${ }^{2021}$ and Buvik et al have demonstrated that video assisted remote clinics have equivalent acceptability to patients compared with F2F consultations. No case of 'missed diagnosis' was recorded in our cohort, although we acknowledge that these consultations were performed for follow-up and this is less likely to occur in this instance.

Ensuring that patients are offered a time interval for their VPC consultation rather than a specific time facilitates greater flexibility. This is useful to clinicians when managing outpatients that may have varied consultation times, be delayed in arrival or require X-ray evaluation during the clinic. It also eliminates long patient waiting times in clinic. Since $28.6 \%$ of written complaints involving hospital and community services in the NHS concern communication and appointments (including delays and cancellations), ${ }^{22}$ effective utilisation of VPC may go a long way in improving the user experience.

VPCs permit healthcare delivery while reducing foot traffic into hospitals, facilitating social distancing and allowing shielding of vulnerable patients. These were strong drivers for their rapid adoption during the COVID-19 pandemic. ${ }^{23}$ They may be conducted in a standalone format or incorporated into existing F2F clinics. The former would facilitate remote working for clinicians and is increasingly valuable in the current climate.

The potential societal cost savings of VPCs are significant. Virtual clinics can be conducted at patients' places of work or home, minimising loss in productivity and improving economy of movement. ${ }^{819}$ They eliminate the need for transport whose cost burden may be borne by the patient, the hospital or the government, depending on how expenses are reclaimed. Moreover, an ecological benefit can be expected since a reduction in travel may result in improved environmental conditions through reduced emissions. ${ }^{24} 25$

Despite the above benefits, there is a danger that the reduced reimbursement of VPCs coupled with the increased use of clinician resources through triaging may disincentivise their widespread use in hospitals. VPC still requires a private space for the consultation and would also require infrastructural investment if video capability is introduced. During the COVID-19 pandemic, CCGs were reimbursing VPCs at F2F rates. However, once the pandemic subsides, if the reimbursement returns to the standard VPC rates, an orthopaedic department could forfeit more than $11.5 \%$ of outpatient clinic reimbursement. We consider various ways to mitigate this. Consultation time may fall as clinicians become more experienced in conducting VPC. Offering patients the choice between VPC or F2F clinics would eliminate the resource spend on screening patients for suitability, but we would have to accept that a proportion of VPC patients would need subsequent F2F if VPC were inadequate. Our unit has begun offering this option and we have found that only 1 in 20 patients opting for VPC require subsequent F2F. We accept that this rate may vary for new referrals as opposed to follow-up consultations. Further solutions may include: (1) diversification of the clinical staff conducting VPCs, such as the addition of a clinical nurse specialist and/or physiotherapist together with a specialist registrar and consultant who are able to 'oversee' consultations and provide support when needed ${ }^{2627}$ and (2) renegotiation of reimbursement for VPCs to equal or outweigh their costs, facilitating their sustainable use and realising the potential societal savings.

One must also consider the influence of the current COVID-19 pandemic on the satisfaction scores from our study. The importance of social distancing and the scale of the pandemic may influence patients and clinicians to rate VPCs higher than they would otherwise. Satisfaction may need to be re-evaluated postpandemic to see whether patient and clinician perceptions of VPC 
change, although we suspect that the roles of VPC will become more refined with time and remain a valuable means of service delivery for a substantial proportion of consultations.

\section{Limitations}

There are several limitations of this study that must be considered when interpreting the findings. First, the relatively small number of patients and clinicians included in this study are limited to elective and fracture clinics involving the subdisciplines of hip, knee foot and ankle surgery, and may therefore, not be representative of other subdisciplines. However, the data obtained from our 23 clinicians compares favourably to previous studies that have reported data on $2-5$ clinicians. ${ }^{10}{ }^{19}$ Second, our cost analysis is based on the duration of consultations and clinician structure of our current orthopaedic outpatient clinics. We accept that this may not represent all clinic structures in the NHS or other parts of the world, but the underlying principles will be relevant. We did not evaluate the reduction in ancillary costs by attending VPC instead of F2F clinics, such as transport costs and time from work, but this has been demonstrated comprehensively by a previous study. ${ }^{23}$ Third, our questionnaires and the labelling of scores $1-2$ as 'dissatisfied' and 3-5 as 'neutral or satisfied' were not validated in this context. However, the questions were based on questions previously utilised in published evaluations of orthopaedic virtual clinics $^{8-10}$ and the use of 5-point Likert scales in this way has been validated. ${ }^{28}{ }^{29}$ Finally, the clinician survey utilised a 5-point Likert scale, while the patient survey used a 10-point Likert scale, which was adjusted to a 5-point scale post-hoc for ease of data reporting. This has been shown to be acceptable with arithmetic conversion. ${ }^{18}$ While we acknowledge these important limitations, we hope to share our experience with virtual clinics in a discipline in which few studies have been published in the hope that other researchers can incorporate these into this rapidly evolving method of service delivery.

\section{CONCLUSION}

While F2F clinics remain the gold standard, VPCs are acceptable to patients and clinicians and play a useful role when clinical examination has already been performed or deemed not to be necessary by the patient or clinician. Offering the choice of VPC or F2F, adding video capability and providing a time interval for the VPC may reduce resources required and increase satisfaction rates. Renegotiating the VPC tariff and cost-saving modifications such as diversifying the clinician structure of the clinic are necessary to ensure sustainability. Further research should be performed on larger study populations to identify patient characteristics that show greatest benefit most from VPCs.

Contributors We hereby confirm that all authors meet the authorship criteria in accordance with the International Committee of Medical Journal Editorial. All of the authors have contributed sufficiently to this work to be listed as authors, and have made substantial contributions to conception and design, or acquisition of data, or analysis and interpretation of data; have been involved in drafting the manuscript and revising it critically for important intellectual content; and have given final approval of the version to be published. Each author has participated sufficiently in the work to take public responsibility for appropriate portions of the content and agreed to be accountable for all aspects of the work. Conception and design: MR, RF and RP. Acquisition of data: RP and SB. Analysis and interpretation of data: CM, SB and WP. Writing original manuscript: WP and RP. Writing-review and editing: MR, $\mathrm{RF}$ and RP.

Funding The authors have not declared a specific grant for this research from any funding agency in the public, commercial or not-for-profit sectors.

\section{Competing interests None declared.}

Patient and public involvement Patients and/or the public were not involved in the design, or conduct, or reporting, or dissemination plans of this research.

\section{Patient consent for publication Not required.}

Ethics approval This study was approved by the Institutional Audit Committee, and since no intervention was performed, Research Ethics Committee approval was not required.

Provenance and peer review Not commissioned; externally peer reviewed.

Data availability statement All data relevant to the study are included in the article or uploaded as online supplemental information. No data are available. All data relevant to the study are included in the article.

Open access This is an open access article distributed in accordance with the Creative Commons Attribution Non Commercial (CC BY-NC 4.0) license, which permits others to distribute, remix, adapt, build upon this work non-commercially, and license their derivative works on different terms, provided the original work is properly cited, appropriate credit is given, any changes made indicated, and the use is non-commercial. See: http://creativecommons.org/licenses/by-nc/4.0/.

\section{ORCID iDs}

Wouter Peeters http://orcid.org/0000-0001-7783-1425

Mark Roussot http://orcid.org/0000-0001-8506-0990

\section{REFERENCES}

1 Haider Z, Aweid B, Subramanian P, et al. Telemedicine in orthopaedics and its potential applications during COVID-19 and beyond: a systematic review. J Telemed Telecare 2020;1357633X:20938241.

2 Bashshur RL. On the definition and evaluation of telemedicine. Telemed J 1995;1:19-30.

3 Pinnock H, Madden V, Snellgrove C, et al. Telephone or surgery asthma reviews? preferences of participants in a primary care randomised controlled trial. Prim Care Respir J 2005;14:42-6.

4 Pinnock H, McKenzie L, Price D, et al. Cost-effectiveness of telephone or surgery asthma reviews: economic analysis of a randomised controlled trial. Br J Gen Pract 2005;55:119-24.

5 Roberts NJ, Partridge MR. Telephone consultations in secondary care. Respir Med 2007;101:1665-9.

6 Beaver K, Tysver-Robinson D, Campbell M, et al. Comparing hospital and telephone follow-up after treatment for breast cancer: randomised equivalence trial. BMJ 2009;338:a3147.

7 Beaver K, Williamson S, Sutton C, et al. Comparing hospital and telephone follow-up for patients treated for stage-I endometrial cancer (ENDCAT trial): a randomised, multicentre, non-inferiority trial. BJOG 2017;124:150-60.

8 Buvik A, Bergmo TS, Bugge E, et al. Cost-effectiveness of telemedicine in remote orthopedic consultations: randomized controlled trial. J Med Internet Res 2019;21:e11330.

9 Buvik A, Bugge E, Knutsen G, et al. Patient reported outcomes with remote orthopaedic consultations by telemedicine: a randomised controlled trial. J Telemed Telecare 2019;25:451-9.

10 Buvik A, Bugge E, Knutsen G, et al. Quality of care for remote orthopaedic consultations using telemedicine: a randomised controlled trial. BMC Health Serv Res 2016;16:483.

11 Kichloo A, Albosta M, Dettloff K, et al. Telemedicine, the current COVID-19 pandemic and the future: a narrative review and perspectives moving forward in the USA. Fam Med Community Health 2020;8.

12 Kruse CS, Krowski N, Rodriguez B, et al. Telehealth and patient satisfaction: a systematic review and narrative analysis. BMJ Open 2017;7:e016242. 
13 Kissler SM, Tedijanto C, Goldstein E, et al. Projecting the transmission dynamics of SARS-CoV-2 through the postpandemic period. Science 2020;368:860-8.

14 Makhni MC, Riew GJ, Sumathipala MG. Telemedicine in orthopaedic surgery: challenges and opportunities. J Bone Joint Surg Am 2020;102:1109-15.

15 Gilbert AW, Billany JCT, Adam R, et al. Rapid implementation of virtual clinics due to COVID-19: report and early evaluation of a quality improvement initiative. BMJ Open Qual 2020;9:e000985.

16 NHS England and NHS Improvement. Clinical guide for the management of remote consultations and remote working in secondary care during the coronavirus pandemic 2020.

17 NHS. NHS improvement 2020/21 national tariff payment system - a consultation notice, 2019. Available: https://improvement.nhs.uk/ resources/national-tariff-2021-consultation/

18 Dawes J. Do data characteristics change according to the number of scale points used? an experiment using 5-point, 7-point and 10-point scales. International Journal of Market Research 2008;50:61-104.

19 Parkes RJ, Palmer J, Wingham J, et al. Is virtual clinic follow-up of hip and knee joint replacement acceptable to patients and clinicians? a sequential mixed methods evaluation. BMJ Open Qual 2019;8:e000502.

20 Greenhalgh T, Wherton J, Shaw S, et al. Video consultations for covid-19. BMJ 2020;368:m998.

21 Car J, Koh GC-H, Foong PS, et al. Video consultations in primary and specialist care during the covid-19 pandemic and beyond. BMJ 2020;371:m3945.
22 NHS Digital. Data on written complaints in the NHS 2017-2018, 2018. Available: https://digital.nhs.uk/data-and-information/ publications/statistical/data-on-written-complaints-in-the-nhs/201718

23 Lanham NS, Bockelman KJ, McCriskin BJ. Telemedicine and orthopaedic surgery: the COVID-19 pandemic and our new normal. JBJS Rev 2020;8:e2000083.

24 Miah S, Dunford C, Edison M, et al. A prospective clinical, cost and environmental analysis of a clinician-led virtual urology clinic. Ann R Coll Surg Engl 2019;101:30-4.

25 Connor MJ, Miah S, Edison MA, et al. Clinical, fiscal and environmental benefits of a specialist-led virtual ureteric colic clinic: a prospective study. BJU Int 2019;124:1034-9.

26 Ferdinandus S, Smith LK, Pandit $\mathrm{H}$, et al. Setting up an arthroplasty care practitioner-led virtual clinic for follow-up of orthopaedic patients. Br J Nurs 2019;28:1326-30.

27 Harding P, Burge A, Walter K, et al. Advanced musculoskeletal physiotherapists in post arthroplasty review clinics: a state wide implementation program evaluation. Physiotherapy 2018;104:98-106.

28 Weijters B, Cabooter E, Schillewaert N. The effect of rating scale format on response styles: the number of response categories and response category labels. International Journal of Research in Marketing 2010;27:236-47.

29 Norman G, scales L. Likert scales, levels of measurement and the "laws" of statistics. Adv Health Sci Educ Theory Pract 2010;15:625-32. 